\title{
On the Solution of a Class of Large Body Problems with Full or Partial Circular Symmetry by Using the Finite-Difference Time-Domain (FDTD) Method
}

\author{
Wenhua Yu, Senior Member, IEEE, Dean Arakaki, Member, IEEE, and Raj Mittra, Life Fellow, IEEE
}

\begin{abstract}
This paper presents an efficient method to accurately solve large body scattering problems with partial circular symmetry. The method effectively reduces the computational domain from three to two dimensions by using the reciprocity theorem. It does so by dividing the problem into two parts: a larger 3-D region with circular symmetry, and a smaller 2-D region without circular symmetry. An finite-difference time-domain (FDTD) algorithm is used to analyze the circularly symmetric 3-D case, while a method of moments (MoM) code is employed for the nonsymmetric part of the structure. The results of these simulations are combined via the reciprocity theorem to yield the radiation pattern of the composite system. The advantage of this method is that it achieves significant savings in computer storage and run time in performing an equivalent 2-D as opposed to a full 3-D FDTD simulation. In addition to enhancing computational efficiency, the FDTD algorithm used in this paper also features one improvement over conventional FDTD methods: a conformal approach for improved accuracy in modeling curved dielectric and conductive surfaces. The accuracy of the method is validated via a comparison of simulated and measured results.
\end{abstract}

Index Terms-finite-difference time-domain (FDTD) methods, method of moments, reciprocity theorem.

\section{INTRODUCTION}

$\mathbf{T}$ HE finite-difference time-domain (FDTD) method is widely applied to simulate electromagnetic propagation [1], [2] because of its versatility and ability to handle complex geometries with arbitrary inhomogeneities. Typically, the spatial discretization used to transform Maxwell's equations into difference equations is on the order of 10 to 20 cells per wavelength at the frequency of interest. Hence, this makes it difficult to apply the FDTD method to large 3-D problems, given the limits of computer storage and run time. However, for a class of geometries that possess azimuthal symmetry, it is possible to reduce the original 3-D problem to an equivalent 2 1/2-D one. This not only makes large problems manageable, but also achieves significant savings in computer storage and run time in the process. This method has been used to model electromagnetic wave scattering [3], subsurface interface radar [4], optical lenses [5], cavity resonance problems [6], and coaxial problems [7]. In many practical applications, the geometry is only partially rather than fully rotationally symmetric. By invoking the reciprocity principle, the method can also be applied to this class of problems, e.g., a paraboloid with a microstrip patch antenna feed [8]. Because the microstrip patch array does not have rotational symmetry, we cannot directly apply the $21 / 2$-D FDTD method to calculate the far zone field of the parabolic antenna system excited by this patch array. However, this asymmetric patch array can be replaced by an equivalent current density calculated by the MoM code. We apply the reciprocity theorem to compute the far zone field of the antenna system excited by this asymmetric source in a manner explained below.

First, we illuminate the antenna system by a plane wave, and use the $21 / 2$-D FDTD method to compute the electric field distribution on the substrate of the patch antenna. Next, we employ the MoM method to derive the current density distribution on the patch array. Finally, we apply the reciprocity theorem to calculate the far zone field of the antenna system excited by an asymmetric source from these known fields and currents.

The reciprocity-based procedure, outlined above, is very useful for solving many 3-D problems belonging to this category, which would otherwise be intractable via the conventional FDTD algorithm when applied directly because of the size of the problem. The purpose of this paper is to address the following issues that arise in the process of adapting the FDTD method to the circularly symmetric case: 1) the singularity problem at the axis of rotation; 2) plane wave source excitation in the cylindrical coordinate system; 3) development of the 2 1/2-D conformal FDTD algorithm for perfect conductors and lossy dielectrics; 4) the procedure for handling an obliquely incident plane wave; and 5) combining FDTD with the reciprocity principle to compute the far-field pattern of a parabolic antenna system.

We choose the example of a reflector antenna illuminated by a plane wave to illustrate the application of the method.

\section{METHOD}

\section{A. FDTD Update Equations for the Axis-Symmetric Case}

To reduce the original 3-D problem and to derive the FDTD update equations for the circularly symmetric case, we begin by expressing the electric and magnetic fields in the cylindrical coordinate system in a Fourier series as:

$$
\vec{E}=\sum_{m=0}^{\infty}\left(\vec{E}_{\text {even }, m} \cos m \phi+\vec{E}_{\text {odd }, m} \sin m \phi\right)
$$




$$
\vec{H}=\sum_{m=0}^{\infty}\left(\vec{H}_{\text {even }, m} \cos m \phi+\vec{H}_{\text {odd }, m} \sin m \phi\right)
$$

where $m$ is the azimuthal harmonic index. Substituting (1) and (2) into Maxwell's equations in the cylindrical coordinate system, we can derive the following FDTD update difference equations:

$$
\begin{aligned}
& E_{\rho}^{n+1}(i, j) \\
& =\frac{\left(1-\frac{\sigma \Delta t}{2 \varepsilon_{r}}\right)}{\left(1+\frac{\sigma \Delta t}{2 \varepsilon_{r}}\right)} E_{\rho}^{n}(i, j)-\frac{\Delta t}{\varepsilon_{r}\left(1+\frac{\sigma \Delta t}{2 \varepsilon_{r}}\right)} \\
& \cdot\left[\frac{H_{\phi}^{n+(1 / 2)}(i, j)-H_{\phi}^{n+(1 / 2)}(i, j-1)}{\Delta z}\right] \\
& -\frac{m \Delta t}{\varepsilon_{r}\left(1+\frac{\sigma \Delta t}{2 \varepsilon_{r}}\right)} \frac{H_{z}^{n+(1 / 2)}(i, j)}{\rho_{i+(1 / 2)}} \\
& E_{\phi}^{n+1}(i, j) \\
& =\frac{\left(1-\frac{\sigma \Delta t}{2 \varepsilon_{r}}\right)}{\left(1+\frac{\sigma \Delta t}{2 \varepsilon_{r}}\right)} E_{\phi}^{n}(i, j)+\frac{\Delta t}{\varepsilon_{r}\left(1+\frac{\sigma \Delta t}{2 \varepsilon_{r}}\right)} \\
& \cdot\left[\frac{H_{r}^{n+(1 / 2)}(i, j)-H_{r}^{n+(1 / 2)}(i, j-1)}{\Delta z}\right] \\
& -\frac{\Delta t}{\varepsilon_{r}\left(1+\frac{\sigma \Delta t}{2 \varepsilon_{r}}\right)} \\
& \cdot\left[\frac{H_{z}^{n+(1 / 2)}(i, j)-H_{z}^{n+(1 / 2)}(i-1, j)}{\Delta z}\right] \\
& E_{z}^{n+1}(i, j) \\
& =\frac{\left(1-\frac{\sigma \Delta t}{2 \varepsilon_{r}}\right)}{\left(1+\frac{\sigma \Delta t}{2 \varepsilon_{r}}\right)} E_{z}^{n}(i, j)+\frac{\Delta t}{\varepsilon_{r}\left(1+\frac{\sigma \Delta t}{2 \varepsilon_{r}}\right)} \\
& \cdot\left[\frac{\rho_{i+(1 / 2)} H_{\phi}^{n+(1 / 2)}(i, j)-\rho_{i-(1 / 2)} H_{\phi}^{n+(1 / 2)}(i-1, j)}{\rho_{i} \Delta \rho}\right] \\
& +\frac{\Delta t}{\varepsilon_{r}\left(1+\frac{\sigma \Delta t}{2 \varepsilon_{r}}\right)} \frac{H_{\rho}^{n+(1 / 2)}(i, j)}{\rho_{i}} \\
& H_{\rho}^{n+(1 / 2)}(i, j) \\
& =H_{\rho}^{n-(1 / 2)}(i, j)-\frac{m \Delta t}{\mu \rho_{i}} E_{z}^{n}(i, j) \\
& +\frac{\Delta t}{\mu}\left[\frac{E_{\phi}^{n}(i, j+1)-E_{\phi}^{n}(i, j)}{\Delta z}\right] \\
& H_{\phi}^{n+(1 / 2)}(i, j) \\
& =H_{\phi}^{n-(1 / 2)}(i, j)-\frac{\Delta t}{\mu}\left[\frac{E_{\rho}^{n}(i, j+1)-E_{\rho}^{n}(i, j)}{\Delta z}\right. \\
& \left.-\frac{E_{z}^{n}(i+1, j)-E_{z}^{n}(i, j)}{\Delta \rho}\right]
\end{aligned}
$$

$$
\begin{aligned}
& H_{z}^{n+(1 / 2)}(i, j) \\
& \quad=H_{z}^{n-(1 / 2)}(i, j)+\frac{m \Delta t}{\mu \rho_{i+(1 / 2)}} E_{\rho}^{n}(i, j) \\
& \quad-\frac{\Delta t}{\mu}\left[\frac{\rho_{i+1} E_{\phi}^{n}(i, j+1)-\rho_{i} E_{\phi}^{n}(i, j)}{\rho_{i+(1 / 2)} \Delta \rho}\right] .
\end{aligned}
$$

In the above equations, the $1 / \rho$ singularity at the axis must be addressed before numerical computations can be performed. There are two general approaches [9] to dealing with this singularity: first, to replace $1 / \rho$ with $d / d \rho$, second, to apply a change of variables $\tilde{E}=\rho E$ and $\tilde{H}=\rho H$. However, these two approaches are not always easy to implement in numerical algorithms. In this paper, we follow a somewhat different procedure that obviates the need for any special treatment of the apparent singularity on the axis for the problem of interest, viz., plane-wave scattering by a reflector antenna.

To generate a normally incident plane wave in the cylindrical coordinate system, we need to set the harmonic number $m$ to 1 . This makes $E_{\rho}$ and $E_{\phi}$ either sine or cosine functions of $\phi$. To handle the singularity on the axis for this $m$, we use Maxwell's finite difference equations (3)-(8). From these equations, we know that only the calculations of $E_{\phi}^{n+1}(1, j)$ and $E_{z}^{n+1}(1, j)$ require the knowledge of $H_{z}^{n+(1 / 2)}(0, j)$ and $H_{\phi}^{n+(1 / 2)}(0, j)$ on the axis. We now use the integral form of Faraday's Law, given by

$$
\oint_{\Delta c} \vec{E} \cdot d \vec{l}=-\oiint_{\Delta s} \mu \frac{\partial \vec{H}}{\partial t} \cdot d \vec{s}
$$

and apply (9) to the region around the $z$-axis. Since the $E_{\phi}$-component is either a sine or cosine function of $\phi$, $\rho_{i+(1 / 2)} \int_{0}^{2 \pi} E_{\phi} \cos \phi d \phi=0$ and, $\rho_{i+(1 / 2)} \int_{0}^{2 \pi} E_{\phi} \sin \phi d \phi=$ 0 , for $m=1$. Hence, it follows that for this $m, H_{z}^{n+(1 / 2)}(0, j)$ is zero on the axis.

The calculation of $H_{\phi}^{n+(1 / 2)}(0, j)$ is a little more involved than it is for $H_{z}^{n+(1 / 2)}(0, j)$; fortunately, it has the $\rho_{i-(1 / 2)}$ factor associated with it in update equation (5). Because the first cell in the $\rho$-direction is a half-cell, $H_{\phi}^{n+(1 / 2)}(0, j)$ always has a zero factor $\left(\rho_{i-(1 / 2)}=0\right)$. As a result, the computation of $H_{\phi}^{n+(1 / 2)}(0, j)$ on the axis is not needed and, hence, the singularity issue becomes moot. Finally, we note from the update equations for the $\phi$-and $z$-polarized electric fields, that neither of these fields are located on the axis; hence, the singularity problem is nonexistent for the computation of these fields.

In order to align the structures with the FDTD grid, we use the nonuniform meshing technique [7]. The first-order Mur boundary condition is employed to truncate the computational domain.

\section{B. Conformal Method for Modeling a Perfect Conductor}

To model a curved surface in the $21 / 2$-D space, we need to deal with the distorted cells (see Fig. 1) in an accurate manner. We follow the procedure described in [10] to handle the distorted cells containing perfect electrical conductor (PEC) boundaries. The magnetic field $H_{\phi}$ corresponding to the distorted cells is computed using Maxwell's integral equations. 


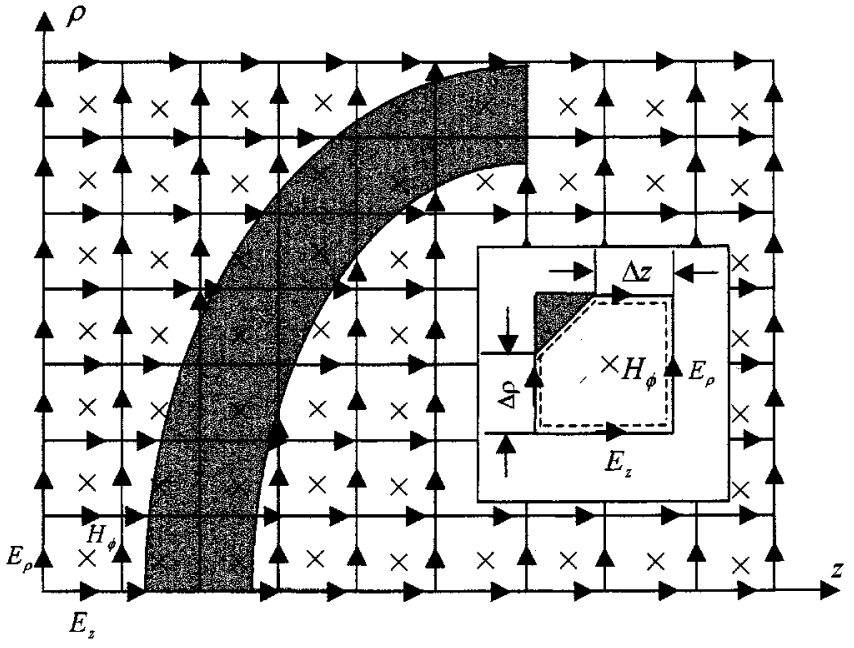

Fig. 1. Conductor with rotational symmetry.

The 2 1/2-D conformal update equation for the $\phi$-polarized magnetic field is given by

$$
\begin{aligned}
H_{\phi}^{n+(1 / 2)}(i, j) \\
=H_{\phi}^{n-(1 / 2)}(i, j)-\frac{\Delta t}{\mu} \\
\cdot\left[\frac{E_{\rho}^{n}(i, j+1) \cdot \Delta \rho(i, j+1)-E_{\rho}^{n}(i, j) \cdot \Delta \rho(i, j)}{\Delta s(i, j)}\right]+\frac{\Delta t}{\mu} \\
\cdot\left[\frac{E_{z}^{n}(i+1, j) \cdot \Delta z(i+1, j)-E_{z}^{n}(i, j) \cdot \Delta z(i, j)}{\Delta s(i, j)}\right]
\end{aligned}
$$

where $\Delta \rho$ and $\Delta z$ are the side lengths of the distorted cell edges that are located in free space, in the $\rho$ and $z$ directions, respectively, and $\Delta s$ is the area of the distorted cell in free space. The conformal update equations of the $H_{\rho}$ and $H_{z}$ are different from those of the 3-D conformal FDTD [10]. The conformal update equations for $H_{\rho}$ and $H_{z}$ in the 21/2-D FDTD can be written as

$$
\begin{aligned}
& H_{\rho}^{n+(1 / 2)}(i, j) \\
& =H_{\rho}^{n-(1 / 2)}(i, j)-\frac{m \Delta t \Delta z(i, j)}{\mu \rho \Delta z_{0}} E_{z}^{n}(i, j) \\
& \quad+\frac{\Delta t}{\mu}\left[\frac{E_{\phi}^{n}(i, j+1)-E_{\phi}^{n}(i, j)}{\Delta z_{0}}\right] \\
& H_{z}^{n+(1 / 2)}(i, j) \\
& =H_{z}^{n-(1 / 2)}(i, j)+\frac{m \Delta t \Delta \rho(i, j)}{\mu \rho_{i+(1 / 2)} \Delta \rho_{0}} E_{\rho}^{n}(i, j) \\
& \quad+\frac{\Delta t}{\mu}\left[\frac{\rho_{i+1} E_{\phi}^{n}(i+1, j)-\rho_{i} E_{\phi}^{n}(i, j)}{\rho_{i+(1 / 2)} \Delta \rho_{0}}\right]
\end{aligned}
$$

where $\Delta \rho_{o}$ and $\Delta z_{o}$ are the side lengths of the regular cells in the $\rho$ - and $z$-directions, respectively. The update equations for the electric fields remain unchanged from the conventional FDTD equations.

\section{Modeling of Curved Dielectric Surfaces}

A slightly different procedure, outlined in [11], is used when the computational domain includes curved dielectric surfaces,

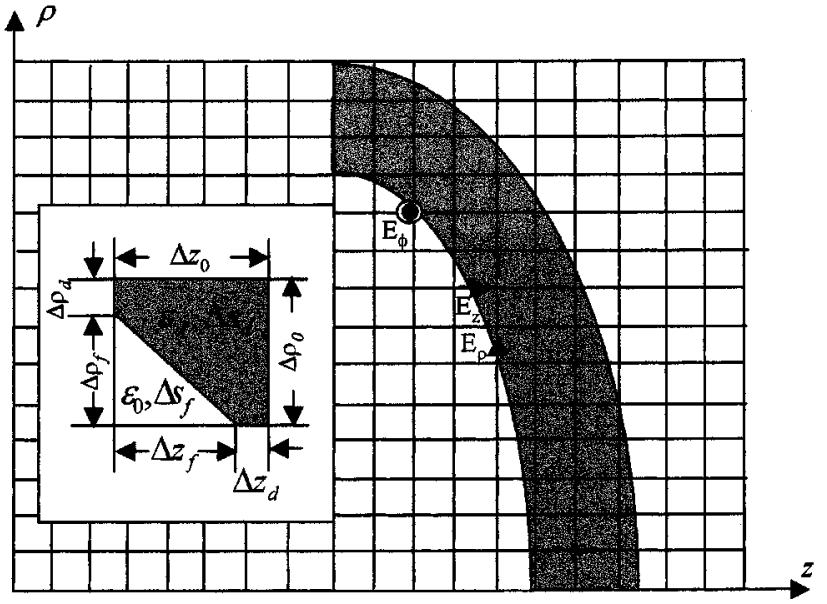

Fig. 2. Dielectric with rotational symmetry.

and it is based on the concept of effective permittivity and conductivity. For the problem at hand, we adapt the algorithm in [11] for the cylindrical coordinate system in $21 / 2-\mathrm{D}$ as follows. We compute the effective permittivity and conductivity by using weighted averages of the distribution of permittivity and conductivity within the distorted cells. For the rotationally symmetric dielectrics considered in this paper, the parameter distribution of the media is uniform in the $\phi$ direction, and the effective permittivity and conductivity of the $\phi$-component in the deformed cell are determined by the intersection of the FDTD lattice and the media in the $\rho-z$ plane. The concept is illustrated in Fig. 2, and the relevant formulas appear below:

$$
\varepsilon_{\mathrm{eff}, \phi}=\frac{\varepsilon_{d} \times \Delta s_{d}+\varepsilon_{0} \times \Delta s_{f}}{\Delta s}
$$

and

$$
\sigma_{\mathrm{eff}, \phi}=\sigma_{d} \frac{\Delta s_{d}}{\Delta s}
$$

where $\varepsilon_{\mathrm{eff}, \phi}$ and $\sigma_{\mathrm{eff}, \phi}$ are the effective permittivity and conductivity of the distorted cells, respectively, and $\Delta s_{f}$ and $\Delta s_{d}$ are the areas of the free space and the dielectric inside the deformed cell, respectively. However, the effective parameters, which correspond to $E_{\rho}$ and $E_{z}$ on the interface between free space and the media, are calculated by the deformed side lengths inside the dielectric and free space. They are written as

and

$$
\varepsilon_{\mathrm{eff}, E z}=\frac{\varepsilon_{d} \times \Delta z_{d}+\varepsilon_{0} \times \Delta z_{f}}{\Delta z}
$$

$$
\begin{aligned}
\sigma_{\mathrm{eff}, E z} & =\sigma_{d} \frac{\Delta z_{d}}{\Delta z} \\
\varepsilon_{\mathrm{eff}, E \rho} & =\frac{\varepsilon_{d} \times \Delta \rho_{d}+\varepsilon_{0} \times \Delta \rho_{f}}{\Delta \rho}
\end{aligned}
$$

and

$$
\sigma_{\mathrm{eff}, E \rho}=\sigma_{d} \frac{\Delta \rho_{d}}{\Delta \rho}
$$

where $\varepsilon_{\mathrm{eff}}, E z$ and $\sigma_{\mathrm{eff}, E z}$ are the effective permittivity and conductivity corresponding to $E_{z}$ on the curved dielectric surface, and $\varepsilon_{\mathrm{eff}, E \rho}$ and $\sigma_{\mathrm{eff}, E \rho}$ corresponding to $E_{\rho}$ on the curved dielectric surface. The subscripts $d$ and $f$ signify dielectric and free space, respectively (see Fig. 2). 


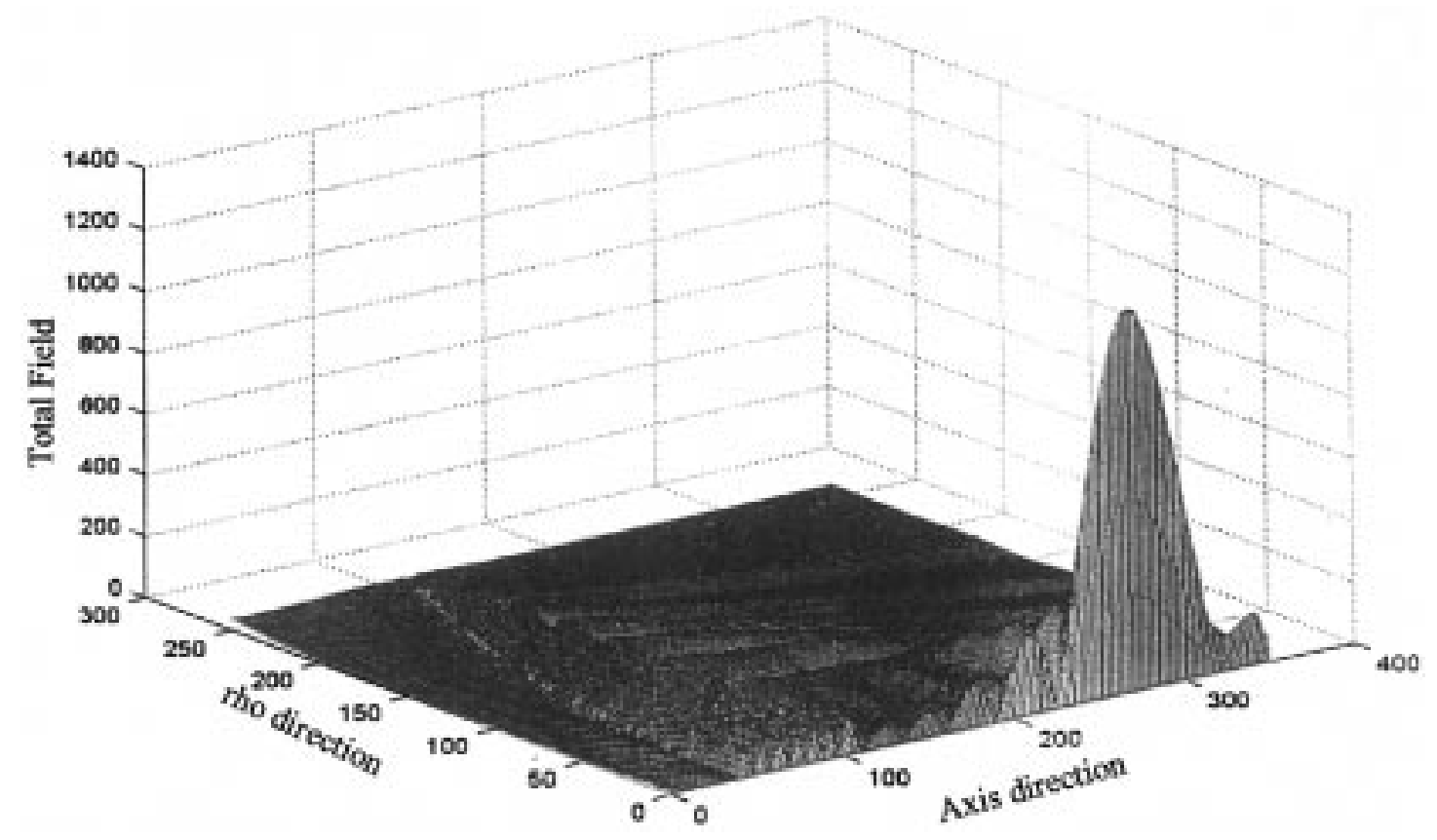

Fig. 3. Global distribution of total field.

\section{Plane-Wave Source Excitation}

In the $21 / 2-\mathrm{D}$ problem, the excitation of a plane wave, with an incident $E_{x}$ (or $E_{y}$ ) field, is accomplished by letting $m=1$. We then have

$$
\begin{aligned}
& E_{\rho}=E_{x 0} \cos \phi \\
& E_{\phi}=-E_{x 0} \sin \phi .
\end{aligned}
$$

Similar equations can be written for the $y$-polarization case, and for the circular polarization as well, by combining the $x$ - and $y$-polarized incident fields with quadrature phasing.

\section{E. Oblique Incidence}

The 2 1/2-D FDTD technique cannot be used to model the case of an obliquely incident plane-wave directly, because it is not rotationally symmetric. To circumvent this problem, we first expand this oblique wave as the superposition of a set of azimuthal harmonics. The singularity treatment at the $z$-axis for the normal incidence plane wave is still valid for the oblique incidence case because we repeatedly model the normal incident harmonic waves instead of an oblique plane wave. Ideally, these harmonic waves should extend to infinity in the $\rho$-direction so that they can reconstruct the original oblique incidence plane wave. Since the FDTD computational domain must necessarily be finite, the tapered source technique is used to realize a smooth transition, from the truncation point to zero, for the last $40 \rho$-cells in the $\rho$-direction. The first-order Mur absorbing boundary condition [12] has been found to be adequate for truncating the boundary of the $21 / 2-\mathrm{D}$ space provided it is placed at a sufficient distance from objects in the FDTD space. In this paper, a normal incidence wave has been extended from the $z$-axis up to 460 cells in the $\rho$-direction. A spline fitting technique has been used to smoothly connect from the truncation point to zero for cell numbers 460 to 500 (boundary of the FDTD domain) in the $\rho$-direction. This smooth transition effectively reduces the effect of mesh truncation in the $\rho$-direction.

We assume that a plane-wave traveling parallel to the $x-z$ plane is obliquely incident upon an antenna system. The electric field can be written as

$$
\vec{E}^{i}=E_{0}\left(\hat{e}_{x} \cos \theta_{i}+\hat{e}_{z} \sin \theta_{i}\right) e^{j\left(-k x \sin \theta_{i}-k z \cos \theta_{i}\right)-j \omega t}
$$

in angular harmonics as follows [13]:

$$
\begin{aligned}
\vec{E}_{x}^{i}= & E_{0} \hat{e}_{x} \cos \theta_{i} e^{-j k z \cos \theta_{i}-j \omega t} \\
& \cdot\left(J_{0}\left(k \rho \sin \theta_{i}\right)+2 \sum_{n=1}^{N} j^{-n} J_{n}\left(k \rho \sin \theta_{i}\right) \cos (n \phi)\right) \\
\vec{E}_{z}^{i}= & E_{0} \hat{e}_{z} \sin \theta_{i} e^{-j k z \cos \theta_{i}-j \omega t} \\
& \cdot\left(J_{0}\left(k \rho \sin \theta_{i}\right)+2 \sum_{n=1}^{N} j^{-n} J_{n}\left(k \rho \sin \theta_{i}\right) \cos (n \phi)\right)
\end{aligned}
$$

where $N$ is determined for a given $\theta_{i}$ to achieve a convergent result $\left(N \cong 5\right.$ for $\theta_{i}=3^{\circ}$ ). We then solve the problem repeatedly for the individual harmonics (different $n$ ), and superimpose the obtained results to construct the solution for the obliquely incident case. The FDTD update equations are extracted from:

$$
\begin{aligned}
\frac{1}{r} & \frac{\partial\left[H_{z} \sin (m \phi)\right]}{\partial \phi}-\frac{\partial\left\lfloor H_{\phi} \cos (m \phi)\right\rfloor}{\partial z} \\
& =\varepsilon \frac{\partial\left[E_{r} \cos (m \phi)\right]}{\partial t}+\frac{1}{2} E_{x 0}(\theta, n) \cos [(n+1) \phi] \\
\frac{1}{r} & \frac{\partial\left[H_{z} \sin (m \phi)\right]}{\partial \phi}-\frac{\partial\left\lfloor H_{\phi} \cos (m \phi)\right\rfloor}{\partial z} \\
& =\varepsilon \frac{\partial\left[E_{r} \cos (m \phi)\right]}{\partial t}+\frac{1}{2} E_{x 0}(\theta, n) \cos [(n-1) \phi]
\end{aligned}
$$

where $E_{x 0}(\theta, n)=E_{0} \cos \theta_{i} J_{n}\left(k \rho \sin \theta_{i}\right)$ and let $m=n-1$ or $n+1$. 


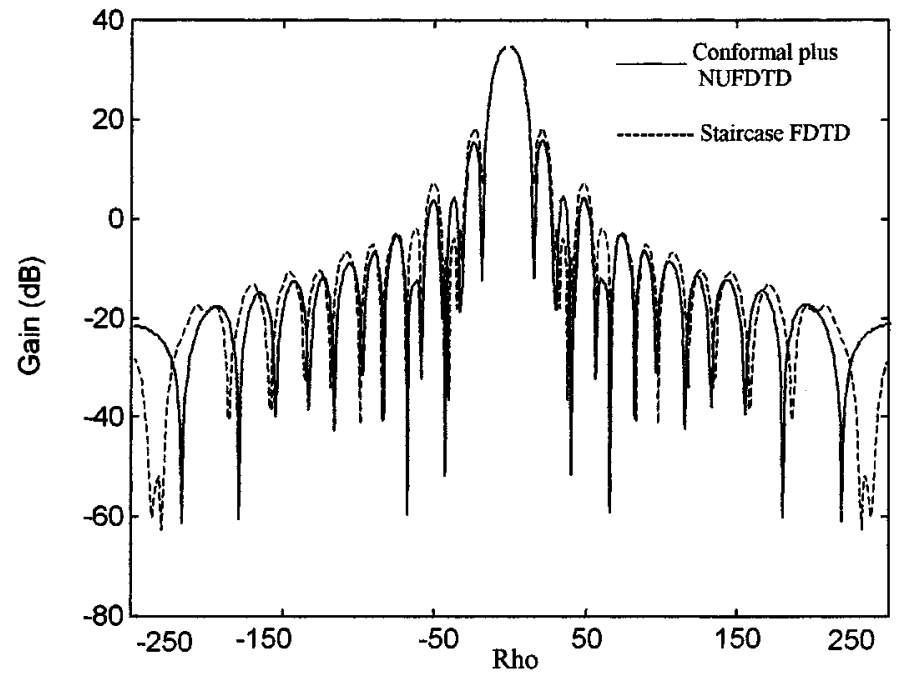

Fig. 4. Comparison between conformal and staircase FDTD results for the main reflector.

\section{F. Feed Analysis}

A microstrip patch antenna is used as the feed of the reflector antenna system. We use an available MoM code, viz., ENSEMBLE, to calculate the current density distribution on the patch array. This current density is used to replace the patch array by using the equivalence theorem. Because meshing of the FDTD computational domain is in the cylindrical coordinate system, and the MoM simulation is in the rectangular coordinate system, we translate the electric field distribution to a rectangular coordinate grid that matches the MoM coordinate points by using a planar interpolation scheme.

\section{G. Reciprocity Theorem}

In this section we briefly explain how the reciprocity theorem is used in our formulation. The reciprocity theorem can be written as

$$
\oint_{S_{1}} \overrightarrow{J_{1}} \cdot \vec{E}_{2} d s=\oint_{S_{2}} \vec{J}_{2} \cdot \vec{E}_{1} d s
$$

where $S_{1}$ is the area of patch array; $\overrightarrow{J_{1}}$ is the current density on the array; $\vec{E}_{1}$, produced by $\vec{J}_{1}$, is the electric field at a far zone point that we are seeking; $S_{2}$ is a far zone plane, where the illuminating electric point dipole source $\vec{J}_{2}$ is located; and $\vec{E}_{2}$ produced by $\vec{J}_{2}$, is the electric field distribution on the surface of the substrate.

We use the MoM-computed current density on the surface of the substrate, the electric field on the surface of the substrate, calculated by the FDTD method, and the known illuminating plane-wave source, to compute the far-field pattern of the antenna system by using the reciprocity theorem as explained in (22). Next, we explain the step-by-step procedure for implementing the reciprocity theorem.

Step 1) Calculate the current densities, $J_{x}$ and $J_{y}$, on the surface of the substrate from the MoM code for a given feed design. These currents replace the patch array by using the Equivalence Theorem.

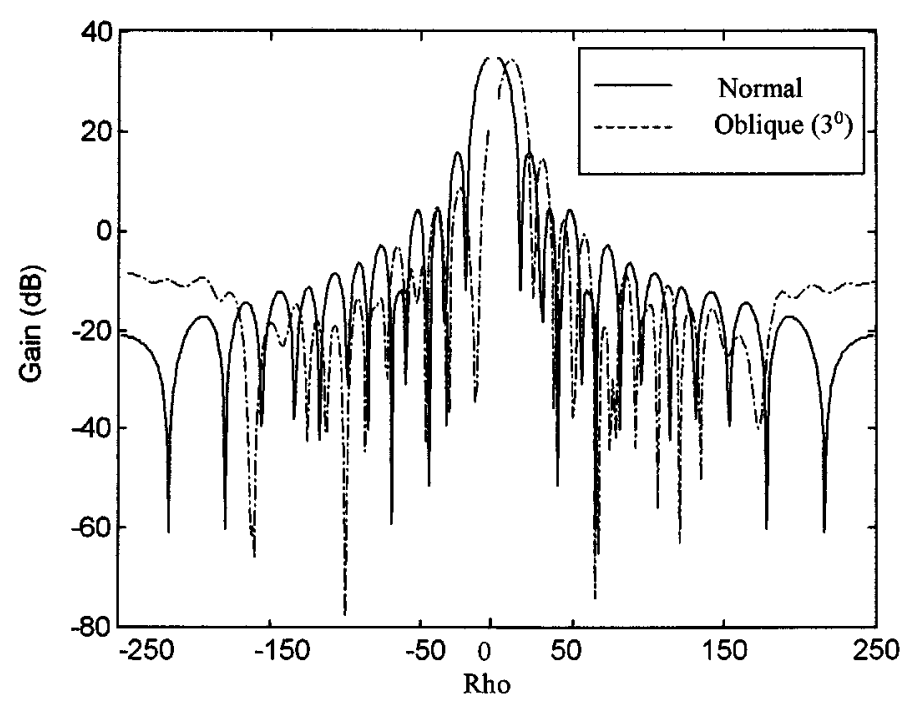

Fig. 5. Comparison between normal and oblique $\left(3^{\circ}\right)$ incidence cases.

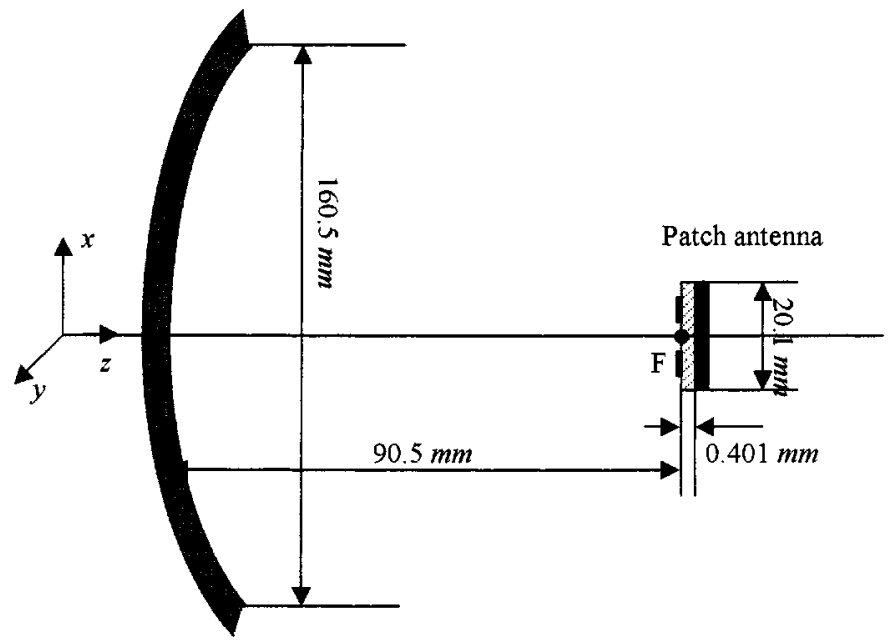

Fig. 6. Geometry of the parabolic antenna and feed.

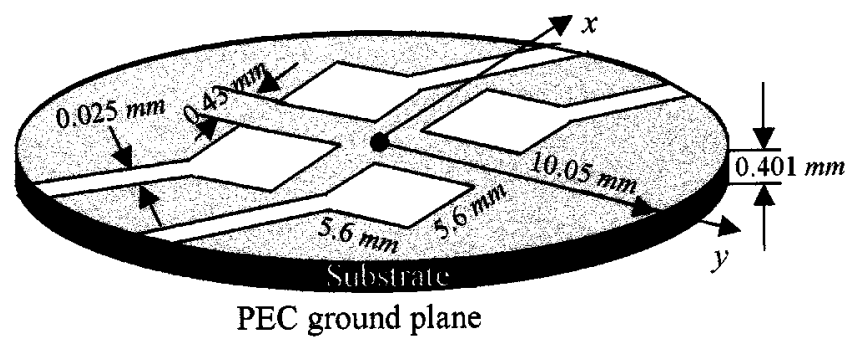

Fig. 7. Geometry of the feed array.

Step 2) Run the FDTD code to calculate the electric field distribution on the surface of the substrate without the patch array.

For the normal incidence case and circularly polarization, the FDTD code is used to calculate the copolarized $\left(E_{x x}, E_{y y}\right)$ and cross-polarized ( $\left.E_{y x}, E_{x y}\right)$ electric field distributions at the location of the patches in the feed array. Note that the fields $\left(E_{x x}\right.$ and $\left.E_{y x}\right)$ and $\left(E_{x y}\right.$ and $\left.E_{y y}\right)$ are generated by $E_{x}$ and $E_{y}$ sources, respectively. The 


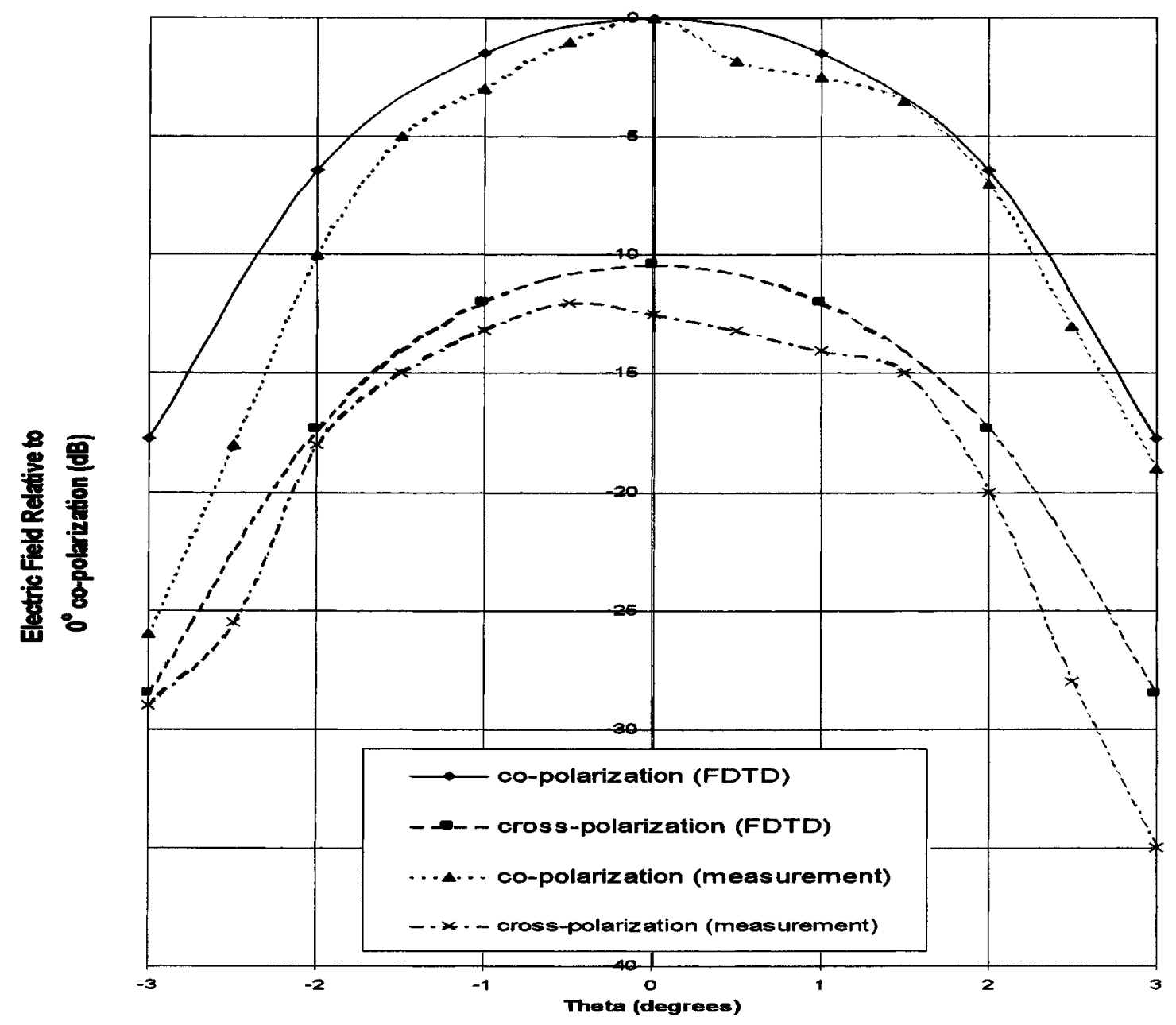

Fig. 8. Comparison with experiment data.

general field $E_{n m}$ represents an $n$-polarized field produced by an $m$-polarized source.

For the oblique incidence case again a circular polarization, repeat the above procedure for the individual harmonics, and superimpose the obtained results to construct the copolarization and cross-polarization fields by using (18).

Step 3) Define the co- and cross-polarization, for circular polarization, for the $x$ - and $y$-directed fields as follows:

$$
\begin{aligned}
E_{x}^{\mathrm{copol}} & =E_{x x}-j \cdot E_{x y}, E_{y}^{\mathrm{copol}} \\
& =E_{y x}-j \cdot E_{y y} \\
E_{x}^{\mathrm{crosspol}} & =E_{x x}+j \cdot E_{x y}, E_{y}^{\mathrm{crosspol}} \\
& =E_{y x}+j \cdot E_{y y} .
\end{aligned}
$$

Step 4) The reciprocity theorem to derive the far-field representation of the antenna system excited by the patch array. Write

$$
\begin{aligned}
& E_{\text {far-zone, copol }} \\
& \quad=\int_{S}\left(E_{x}^{\mathrm{copol}} \cdot J_{x}+E_{y}^{\mathrm{copol}} \cdot J_{y}\right) d S
\end{aligned}
$$

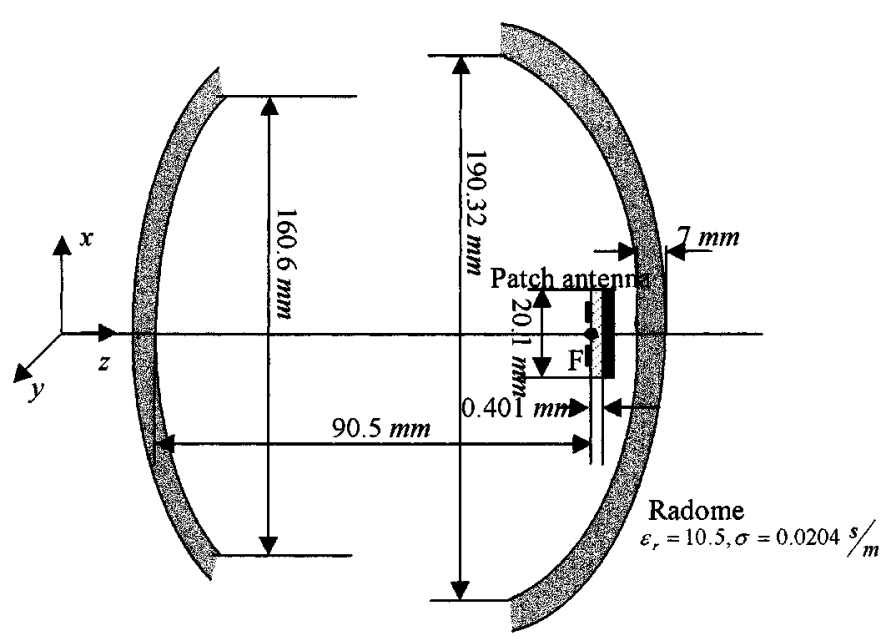

Fig. 9. Geometry of the antenna system including the main reflector and feed.

$$
\begin{aligned}
& E_{\text {far-zone, crosspol }} \\
& \quad=\int_{S}\left(E_{x}^{\mathrm{crosspol}} \cdot J_{x}+E_{y}^{\mathrm{crosspol}} \cdot J_{y}\right) d S
\end{aligned}
$$

where $S$ is the surface of the radiating patches. 


\section{Co- and Cross-pol Values vs. Incident Angle}

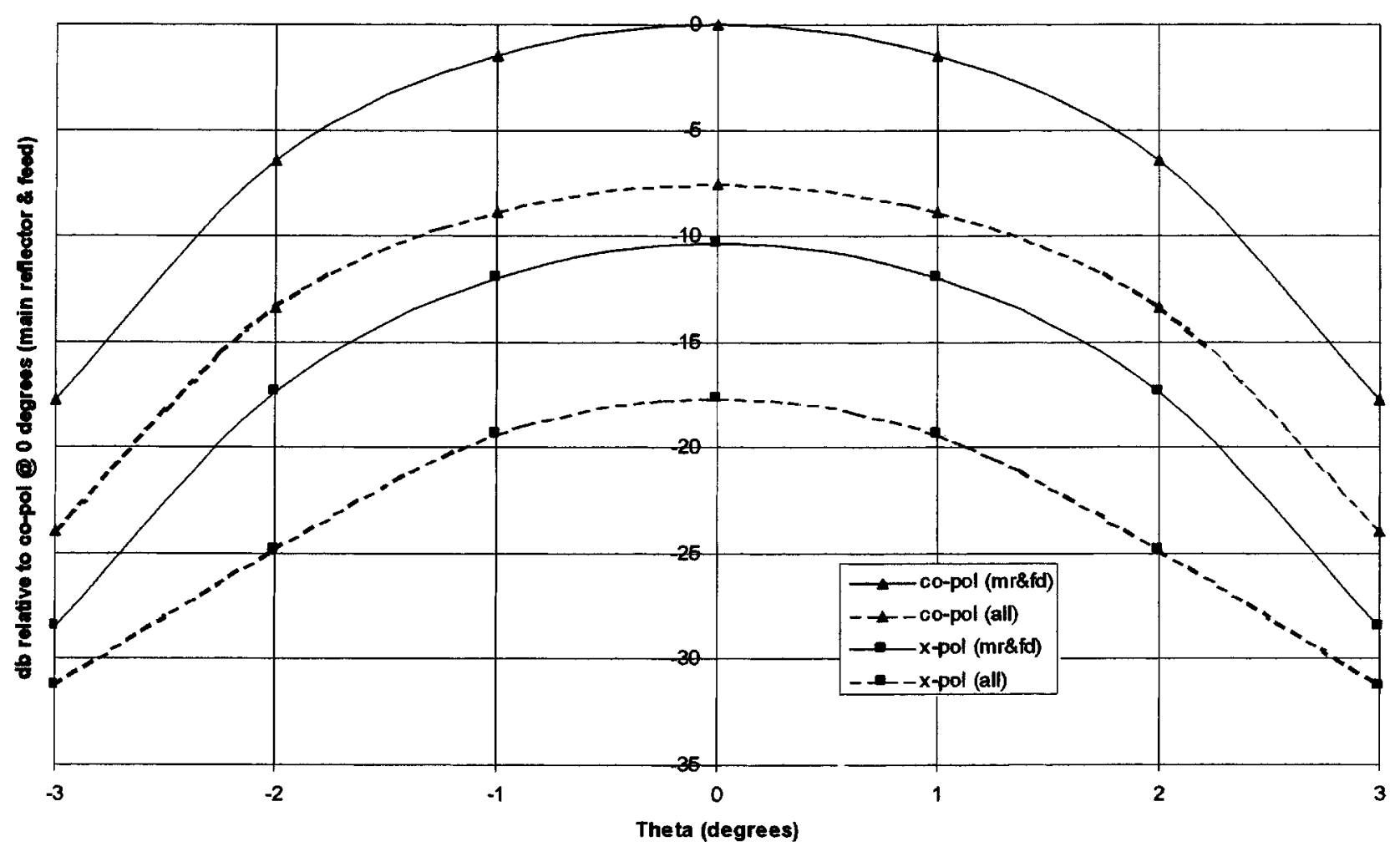

Fig. 10. Co- and cross-polarization gains versus incident angle.

Step 5) Calculate the gain of the system from the ratio of this field intensity to that produced by an isotropic radiator.

$$
\text { Gain }=20 \times \log \left(\frac{E_{\text {system }}}{E_{\text {reference }}}\right) .
$$

Step 6) Calculate the far zone field pattern first by changing direction of illuminator of the incident plane wave and then rotating the feed structure about the $z$-axis by the desired angular displacement. Note that the 2 1/2-D FDTD simulation cannot be rotated in the $\phi$-direction since it is rotationally-invariant in this direction.

Repeat the procedure above for other incidence plane angles, to compute entire pattern of the antenna system.

\section{NUMERICAL RESULTS}

To illustrate the procedure described in the last section, we calculate the field distribution in the focal region of a paraboloidal reflector antenna. The antenna has an aperture diameter of $160.5 \mathrm{~mm}(24.07 \lambda)$, focal length of $90.5 \mathrm{~mm}$ (13.58 $\lambda$ ), and an operating frequency of $45 \mathrm{GHz}$. We first calculate $E_{\rho}$ and $E_{\phi}$ from the FDTD program, then obtain $E_{x}, \vec{E}_{x}=\hat{\rho} E_{\rho} \cos \phi-\hat{\phi} E_{\phi} \sin \phi$. Fig. 3 presents the global field distribution of the reflector for the normal incidence case, obtained by using the conformal FDTD approach discussed in the last section. The radial-field distributions are plotted in
Fig. 4, for the staircase- and conformal-FDTD methods for comparison. In both computations, $\Delta z$ and $\Delta \rho$ equal one-twentieth of the operating wavelength. The total computational domain includes $500 \times 340$ cells.

Second, we consider an obliquely incident plane-wave illuminating the reflector at an angle of $3^{\circ}$ with respect to the $z$-axis. Fig. 5 shows the comparison between the normal and oblique incident cases. Note that, as expected, the maximum of the focal region field shifts $3^{\circ}$ in the direction opposite to the shift of the incident plane wave.

Next, to illustrate the applicability of the approach to geometries with only partial circular symmetry, we introduce a microstrip feed (see Figs. 6 and 7), in the focal region of the reflector, which is illuminated by a circularly polarized plane wave, with incident angles of $1^{\circ}, 2^{\circ}$, and $3^{\circ}$. The patch antenna array, obviously, does not possess circular symmetry. The gain values plotted in Fig. 8 are ratios $(\mathrm{dB})$ of the far-zone electric fields, for various incidence angles, to the copolarized far-zone electric field at $0^{\circ}$ incidence. The figure compares the simulated and measured results and the two are seen to be in fairly good agreement.

The asymmetric behavior of the far-zone field pattern can be determined by the current density distribution on the patch array. The simulation results correspond to the plane defined by $\phi=0^{\circ}$ and the various $\theta$ values plotted in Fig. 8. For patterns in other planes, the feed structure requires rotation about the $z$-axis by the desired angular displacement. Note that the 2 1/2-D FDTD simulation cannot be rotated in the $\phi$-direction since it is rotationally invariant in this direction. 
The final example is the above antenna covered by a radome that has an aperture diameter of $190.32 \mathrm{~mm}(28.55 \lambda)$, shown in Fig. 9. The data plotted in Fig. 10 are ratios $(\mathrm{dB})$ of the far-zone electric fields, for various incidence angles, to the copolarized far-zone electric field at $0^{\circ}$ incidence.

Though not included here, we have also considered the case where the radome may not be strictly circularly symmetric.

\section{CONCLUSION}

This paper has presented an efficient method for determining radiation patterns for electrically large scatterers with partial circular symmetry. Through the use of the reciprocity theorem, a significant reduction in computer resource requirements has been achieved. We have discussed the conformal method for modeling curved dielectric and conducting surfaces, and the tapered source method for obliquely incident plane-wave excitation. FDTD update equations were also derived for obliquely incident plane waves. The overall method was verified by comparing simulated results, for both normal and oblique incidence cases to measured data; and the two were found to be in good agreement. Thus, for a class of partial axisymmetric geometries, significant improvement in computational efficiency can be attained through the use of this method, without sacrificing calculation accuracy.

\section{REFERENCES}

[1] K. S. Yee, "Numerical solution of initial boundary value problems involving Maxwell's equations in isotropic media," IEEE Trans. Antennas Propagat., vol. AP-14, pp. 302-307, May 1966.

[2] S. Chebolu, S. Dey, R. Mittra, and J. Svigelj, "Efficient modeling of microstrip antennas using the finite-difference time-domain method," in Advances in Microstrip and Printed Antennas, K. F. Lee and W. Chen, Eds. New York: Wiley, 1997.

[3] C. L. Bitt, "Solution of electromagnetic scattering problems using time domain techniques," IEEE Trans. Antennas Propagat., vol. 37, pp. $1181-1192,1989$.

[4] M. E. Moghaddam, J. Yannakakis, and W. C. Chew, "Modeling of the subsurface interface radar," J. Electromagn. Waves Applicat., vol. 5, no. 1, pp. 17-39, 1991.

[5] D. B. Davidson and R. W. Ziolkoski, "Body of revolution finite-difference time-domain modeling of space time focusing by a three dimensional lens," J. Opt. Soc. Amer., June 1993.

[6] Y. Chen and R. Mittra, "Finite-difference time-domain algorithm for solving Maxwell's equations in rotationally symmetric geometries," IEEE Trans. Microwave Theory Tech., vol. 44, pp. 832-839, June 1996.

[7] W. Yu and R. Mittra, "A technique for improving the accuracy of the nonuniform finite difference time domain (FDTD) algorithm," IEEE Trans. Microwave Theory Tech., vol. 47, pp. 353-356, Mar. 1999.

[8] R. Mittra, S. Dey, S. Chakravarty, and N. V. Veremey, "Reciprocity approach to pattern computation of a microstrip antenna operating in a complex environment," in Proc. IEEE AP-S Int. Symp., vol. 2, Atlanta, GA, June 1998, pp. 1138-1140.

[9] B. Yang, D. Gottlieb, and J. S. Heshaven, "On the use of PML ABC's in spectral time-domain simulations of electromagnetic scattering," in Proc. Appl. Computat. Electromagn., CA, Mar. 1997, pp. 926-933.

[10] S. Dey and R. Mittra, "A locally conformal finite difference time domain (FDTD) algorithm for modeling 3-D objects with curved surfaces," in Proc. IEEE AP-S Int. Symp., Montreal, Canada, 1997.

[11] N. Kaneda, B. Houshmand, and T. Itoh, "FDTD analysis of dielectric resonators with curved surfaces," IEEE Trans. Microwave Theory Tech., vol. 45, pp. 1645-1649, Sept. 1997.

[12] G. Mur, "Absorbing boundary conditions for the finite-difference approximation of the time-domain electromagnetic field equations," IEEE Trans. Electromagn. Compat., vol. 23, no. 3, pp. 377-382, 1981.

[13] C. A. Balanis, Advanced Engineering Electromagnetics. New York: Wiley, 1989, pp. 614-618. 